\title{
Lattice-Image Estimates of Nano-Particle Fraction-Crystalline
}

\author{
P. Fraundorf ${ }^{1}$, Somik Mukherjee ${ }^{2}$ \\ 1. Center for NanoScience/Physics \& Astronomy, University of Missouri StL, St. Louis MO 63121 USA \\ 2. Electrical and Computer Engineering, University of Missouri, Columbia MO 65211 USA
}

The challenge of estimating the crystalline-fraction of dense randomly-oriented nano-particles, contained in a (less-dense) amorphous support, is increasingly important in fields as diverse as heterogeneous-catalyst design and electronic-data storage. Lattice-fringe visibility-analysis [1,2] is a potential tool in this context, but of course interfering contrast from the amorphous support (as well as lack of crystalline order) might contribute to the disappearance of detectable fringes in sufficiently small particles.

In this paper we describe a lattice-fringe visibility-model strategy for estimating "fraction-crystalline". In that model, the amorphous-support complicates estimation of a cluster's shape-transform detectability-threshold $\Gamma / t$ (in spatial-frequency or excitation-error units) whose reciprocal $t / \Gamma$ measures effective-thickness when modeling lattice-fringe visibility-band widths. Our trick is to reparameterize using one parameter (single/cross-fringe ratio) that is independent of fraction-crystalline, and another (no-fringe-fraction) that is proportional to it. Constraints on $t / \Gamma$ help, but aren't required.

The first step in measurement is to define criteria for: (i) identifying nano-particles of a given size in the support (e.g. by virtue of the shape and size of their excess scattering density) and (ii) determining if an identified nano-particle is showing evidence for zero, one, or more than one lattice-fringe e.g. by peakdetection in power spectra of small images centered on each particle candidate as shown in Fig. 1. The possibility of automating such processes is illustrated in Fig. 2, with a test array of simulated particleimages pasted into an $8 \times 8$ array. This latter process, on experimental data, is still a work in progress.

The second step is to experimentally identify and categorize a subset of randomly-oriented particles e.g. from a single HRTEM image of their amorphous support. Assume contrast transfer for at least (111) \& (200) fringes. If particles are sometimes found to have more than one crystal e.g. as a result of twinning, you might consider only the largest lattice in each. In effect, you thereby obtain data on $\mathrm{N}$ particles of which $\mathrm{N}_{0}$ evidenced no fringes, $\mathrm{N}_{1}$ evidenced one only, and $\mathrm{N}_{\mathrm{x}}$ evidenced two or more i.e. cross-fringes.

Work reported elsewhere in the proceedings (on geometry vs. paint models of fcc lattice-fringe visibility) has given us approximate analytical as well as numerical models of single/cross-fringe ratio and no-fringe-fraction. On the plot of these quantities in Fig. 3, the models define a trajectory insensitive to electron-energy, lattice-parameter, and even detectability of (220) fringes, parameterized by the (perhaps unknown) effective thickness $t / \Gamma$.

This allows one to estimate $t / \Gamma$ and the no-fringe-fraction $f_{0}$ from the single-to-cross fringe fraction $f_{1} / f_{x}$ $\approx \mathrm{N}_{1} / \mathrm{N}_{\mathrm{x}}$. The estimated fraction-crystalline is the observed value of $\mathrm{N}_{0} / \mathrm{N}$ divided by the predicted value $\mathrm{f}_{0}$ for an all-crystalline set. Fig. 4 illustrates the same quantities on a ternary plot. More study is needed to determine the accuracy of this approach. Analysis of ad-hoc data from this study [3], however, suggests that a non-crystalline fraction may be detectable even when small cluster-sizes mean contrast from the non-crystalline support is significant, and noise-parameter $\Gamma$ becomes difficult to model. 


\section{References:}

[1] P. Fraundorf et al. (2005) Journal of Applied Physics 98:114308

[2] P. Wang et al. (2006) Ultramicroscopy 106:277-283.

[3] S. Mukherjee et al. (2012) Nanotechnology 23:485405.

[4] Thanks to Missouri nano-alliance participants for a continuous stream of interesting challenges.
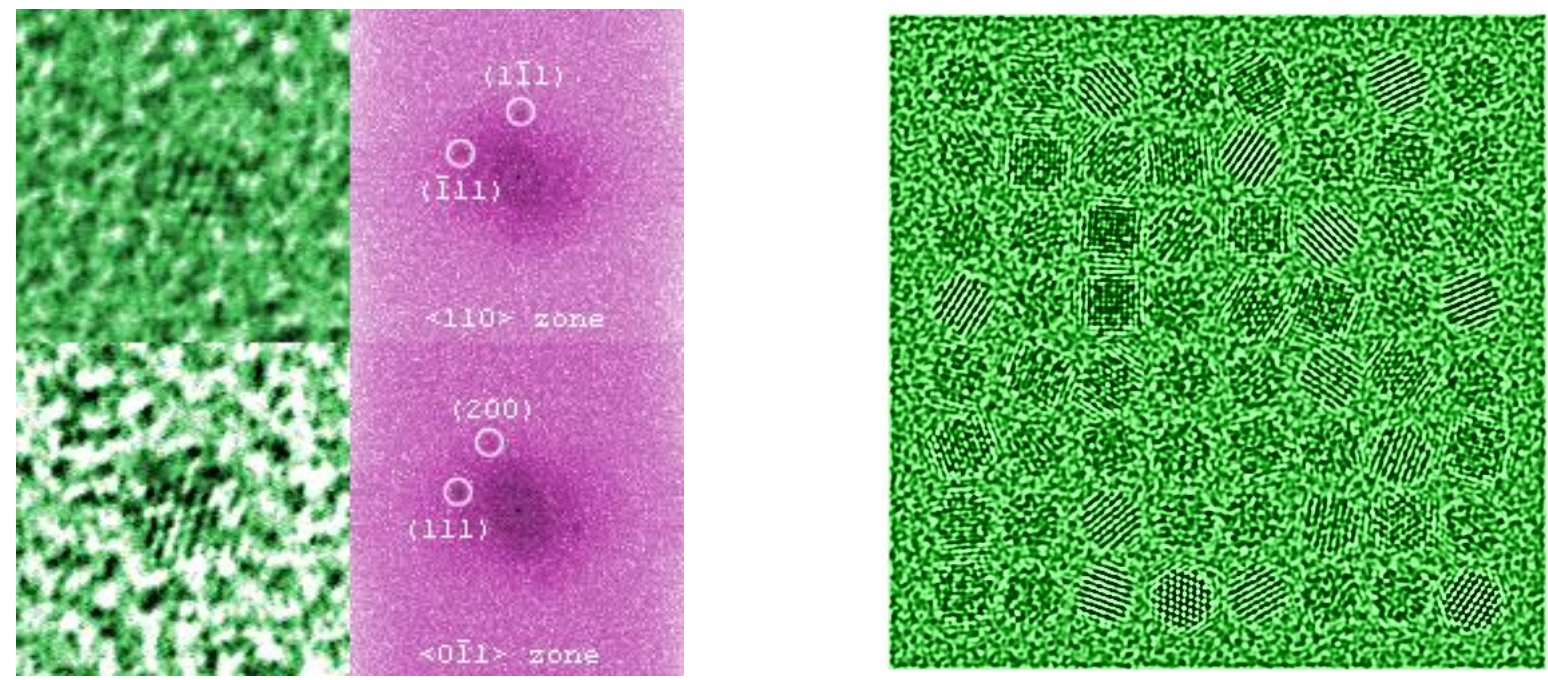

Figure 1. Fringed fcc-Pt nanoparticles in a non-crystalline matrix.

Figure 2. Simulated 8x8 array of randomly-oriented nanoparticles for automated analysis testing. fec lattice-fringe visibility-model
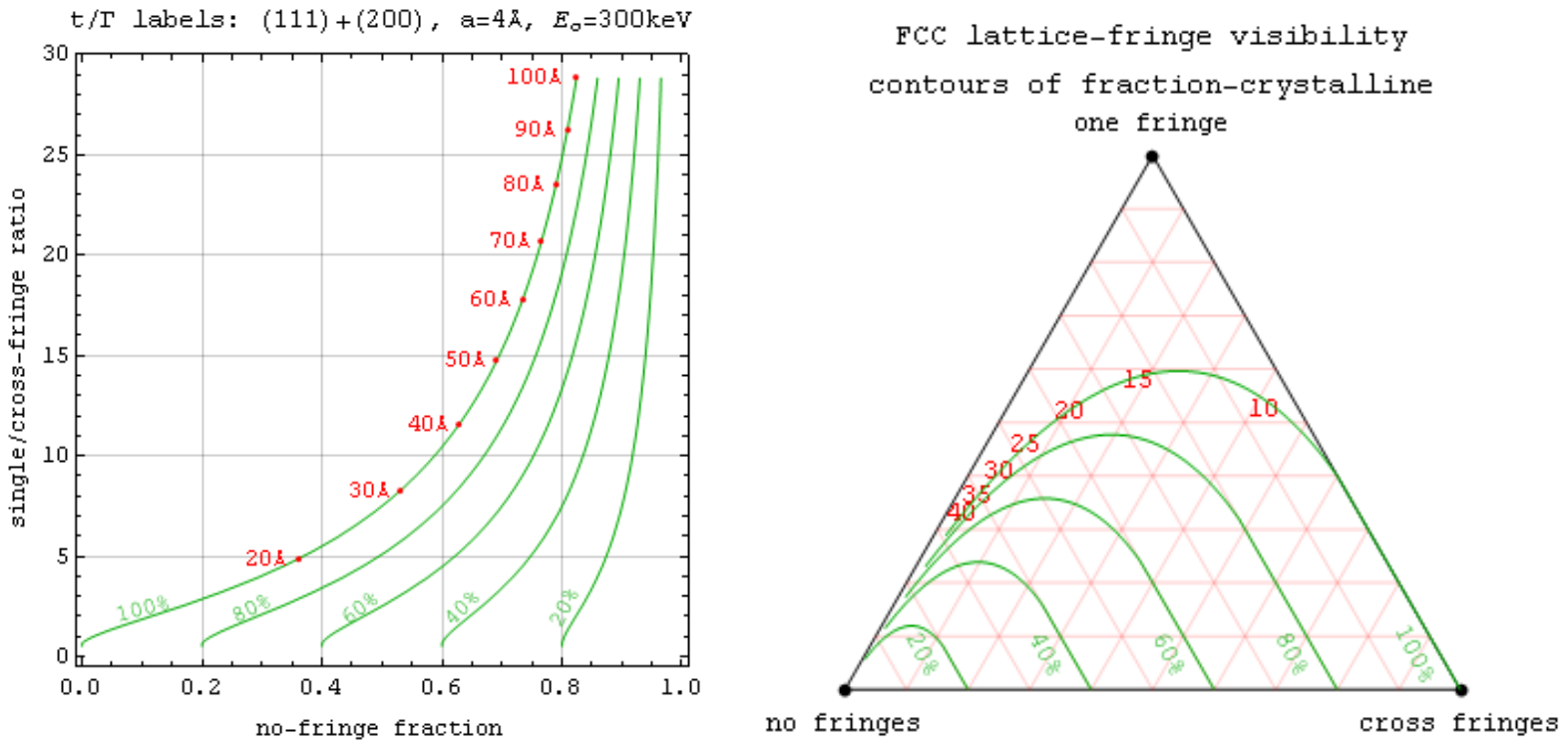

Figure 3. Single/cross-fringe ratio vs no-fringe-fraction fraction-crystalline isocontours.

Figure 4. Same isocontours (and same $t / \Gamma$ values in red) on a ternay diagram of $f_{0}, f_{1}$ and $f_{x}$. 